\title{
Acetylator Status and Diabetic Neuropathy
}

\author{
G. M. Shenfield, V.J. McCann and R. Tjokresetio \\ Department of Pharmacology, University of Western Australia and Diabetes Clinic, Royal Perth Hospital, Perth, Western Australia
}

\begin{abstract}
Summary. Acetylator status was determined in 112 normal and 116 diabetic subjects. Forty-eight percent of the normal subjects were fast acetylators compared with $74 \%$ of the Type 1 (insulin-dependent) diabetic and $54 \%$ of the Type 2 (non-insulin-dependent) diabetic subjects. This result for Type 1 diabetic patients was significantly different from the normal subjects, while that for Type 2 patients was intermediate between the two. In contrast to a previous report, there was no significant association of acetylator status with peripheral neuropathy in subjects with either Type 1 or Type 2 diabetes. Acetylator status could be a genetic marker for Type 1 diabetes, but the increased proportion of fast acetylators in both groups suggested the possibility of an artefact due to high glucose levels. The rate of acetylation of sulphadimidine was significantly greater in both fast and slow acetylators with diabetes compared with normal subjects and there was a significant association between high plasma glucose and acetylator status. Prospective studies will be necessary to confirm whether acetylator status is a true genetic marker for diabetes.
\end{abstract}

Key words: Acetylation, genetics of diabetes, hyperglycaemia, neuropathy, Type 1 and Type 2 diabetes.

\footnotetext{
A number of drugs are metabolised in the liver by the enzyme $\mathrm{N}$-acetyltransferase [1]. The rate of activity of this enzyme is genetically determined and populations may be divided into 'slow' and 'fast' acetylators with implications for the dosage requirements of a variety of drugs [2]. The total $\mathrm{N}$-acetyltransferase activity present in the liver of a rapid acetylator is at least twice as high as that found in a slow acetylator [3] and there is considerable racial variation with most Caucasoid populations having approximately 50\% of their number in each group [2]. Slow acetylators are
}

more prone than fast acetylators to develop peripheral neuropathy when treated with isoniazid $[4,5]$ and a study from Finland found that seven out of nine Type 1 (insulin-dependent) diabetic children were fast acetylators [6]. McLaren et al. therefore investigated acetylator status in relation to diabetic neuropathy [7]. They found that diabetic patients without peripheral neuropathy had a significantly higher proportion of fast acetylators than a control group. Thus fast acetylation appeared to 'protect' against neuropathy.

Burrows et al. [8] in a much smaller group of nonselected diabetic patients found an increased prevalence of fast acetylators, but numbers were too small to assess relative states of neuropathy. They suggested that McLaren's data could be explained if a) fast acetylators are more susceptible to diabetes than slow acetylators and b) slow acetylators with diabetes are more susceptible to neuropathy. If the first statement is true, then acetylator status might be an additional genetic marker for diabetes. Bodansky et al. [9] combined the data from previous studies $[6,7]$ with those from 55 Type 1 diabetic patients from their own clinic and found an increased frequency of fast acetylators. They suggested that fast acetylator phenotype is a genetic marker for Type 1 diabetes and that more than one genetic locus may therefore be involved in susceptibility to Type 1 diabetes.

The present study was designed to investigate the association between acetylator status and peripheral neuropathy in both Type 1 and Type 2 diabetic patients.

\section{Patients and Methods}

The study was approved by the Ethics Committee of Royal Perth Hospital and 112 normal and 116 diabetic subjects were investigated. There were 47 Type 1 and 69 Type 2 diabetic patients. The latter 
Table 1. Acetylator status of normal and diabetic subjects

\begin{tabular}{lllrl}
\hline & Fast & Slow & Total & $p^{\text {a }}$ \\
\hline Normal subjects & $54(48 \%)$ & $58(52 \%)$ & 112 & - \\
Type 1 diabetes & $35(74 \%)$ & $12(26 \%)$ & 47 & $<0.01$ \\
Type 2 diabetes & $42(61 \%)$ & $27(39 \%)$ & 69 & NS \\
Types 1 and 2 diabetes & $77(66 \%)$ & $39(34 \%)$ & 116 & $<0.01$ \\
\hline
\end{tabular}

${ }^{a}$ Compared with normal subjects; NS $=$ not significant

Table 2. Acetylator status in relation to neuropathy

\begin{tabular}{lrrllll}
\hline & \multicolumn{2}{c}{ Type 1 diabetes } & & \multicolumn{2}{c}{ Type 2 diabetes } & \\
\cline { 2 - 3 } & Fast & Slow & & Fast & Slow & Total \\
\hline Total & 35 & 12 & & 42 & 27 & 116 \\
Neuropathy & 9 & 5 & & 19 & 16 & 49 \\
No neuropathy & 26 & 7 & & 23 & 11 & 67 \\
\hline
\end{tabular}

Table 3. Random clinic plasma glucose level and glycosylated haemoglobin level for 1 year preceding study

\begin{tabular}{|c|c|c|c|}
\hline & \multicolumn{3}{|c|}{ Plasma glucose $(\mathrm{mmol} / \mathrm{l})$} \\
\hline & Type 1 diabetes & Type 2 diabetes & $p$ \\
\hline Fast & $12.5 \pm 0.75$ & $13.2 \pm 0.60$ & NS \\
\hline Slow & $10.6 \pm 0.92$ & $11.2 \pm 0.68$ & NS \\
\hline \multirow[t]{2}{*}{$p$} & NS & $<0.05$ & \\
\hline & \multicolumn{3}{|c|}{ Glycosylated haemoglobin (\%) } \\
\hline Fast & $11.4 \pm 1.7$ & $11.1 \pm 2.2$ & NS \\
\hline Slow & $10.6 \pm 1.3$ & $11.3 \pm 2.2$ & NS \\
\hline$p$ & NS & NS & \\
\hline
\end{tabular}

Results expressed as mean $\pm \mathrm{SEM}$; NS $=$ not significant

group presented over the age of 40 years and 26 of them had always been treated with diet alone or oral hypoglycaemic agents. Fortythree patients had been initially controlled with diet or oral therapy but because of poor control had subsequently been transferred to treatment with insulin. The mean ( \pm SEM) ages of the patients were: Type 1, $44.2 \pm 2.3$ years and Type $2,63.5 \pm 1.3$ years. The known mean duration of diabetes was respectively: Type 1,14.2 \pm 1.5 years and Type 2,10.5 \pm 1.1 years. The normal and diabetic subjects were all Caucasoids of European descent. An Australian population contains more individuals of Southern European origin than would a British control group. The diabetic patients were selected on the basis of the presence or absence of neuropathy which had been identified for a previous study [10]. It was defined as neurological symptoms together with loss of touch or proprioceptive sensation in the lower limbs, muscle wasting and weakness accompanied by loss of tendon reflexes or two or more of the features of autonomic neuropathy. Loss of vibration sense or tendon reflexes alone in elderly patients were not accepted as evidence of neuropathy.

Acetylator status was measured by the method of Schröder [11] in which a standard dose of sulphadimidine is taken after an overnight fast and a 5 $-6 \mathrm{~h}$ urine specimen assayed colorimetrically for total and free sulphadimidine. No patients with renal failure were included. Those with less than $70 \%$ of the drug as the acetylated metabolite were classified as slow acetylators, the rest as fast acetylators.
Glycosylated haemoglobin levels were estimated by column chromatography using mini-columns as described previously [10]. Statistical analysis was performed by Student's unpaired 't'-test and the $\chi^{2}$ test.

\section{Results}

Table 1 gives the acetylator status for the normal subjects and the two groups of diabetic patients. The prevalence of fast acetylators was significantly greater in the Type 1 diabetic subjects than in the normal subjects. Although increased in the Type 2 group, difference in prevalence was not significant. If all diabetic patients are combined, there was also a significant increase in the percentage of fast acetylators compared with the healthy subjects $(p<0.01)$. The mean $( \pm$ SEM) percentage acetylation of the drug for the healthy group was $81.5 \pm 0.9 \%$ (fast) and $49.5 \pm 1.9 \%$ (slow). For all diabetic patients, it was $85.1 \pm 0.9 \%$ (fast) ( $p<0.05$ compared with normal subjects) and $59.0 \pm 1.9 \%$ (slow) $(p<0.001$ compared with normal subjects). For the Type 1 diabetic group, the mean percentage acetylation was $84.5 \pm 1.5 \%$ (fast) and $59.6 \pm 2.8 \%$ (slow). This was significantly different from the healthy group $(p<0.01)$ but not significantly different from other diabetic patients.

Forty-nine patients had neuropathy and Table 2 indicates their distribution in the two groups of patients in relation to acetylator status. There was no significant difference in the proportion of fast acetylators in those with neuropathy compared with those without neuropathy in either group of patients.

Mean random clinic plasma glucose levels (usually $2 \mathrm{~h}$ post-prandial) and glycosylated haemoglobin for the year preceding the present study are shown in Table 3. The mean plasma glucose level was higher in fast than slow acetylators in Type 1 diabetic subjects but the difference did not achieve statistical significance. In Type 2 patients, the mean glucose was significantly higher in fast acetylators $(p<0.05)$. The mean glycosylated haemoglobin level was not significantly different in either group of subjects.

\section{Discussion}

We have therefore found a significant increase in fast acetylators with Type 1 diabetes and in Type 2 diabetes with poor diabetic control. This was the case in spite of the fact that, in our control population, the prevalence of fast acetylators (48\%) was considerably higher than that reported for the British population (37\%) [12]. In contrast to the results of McLaren et al. [7], there was no significant association between acetylator status and diabetic neuropathy. 
Our findings on the prevalence of fast acetylators are in agreement with those of Burrows et al. [8] and Bodansky et al. [9]. If it is accepted that there is a primary association between Type 1 diabetes and fast acetylation, then this may be evidence of another genetic marker for this type of diabetes. It is known that slow acetylators are autosomal, homozygous recessives [13] but it is not known which chromosome carries the responsible genes. It may therefore be a quite independent marker from others of Type 1 diabetes such as HLA status or properdin factor $[14,15]$.

Burrows et al. [8] suggested that there might also be an association between some Type 2 diabetic patients and acetylator phenotype and it is possible that a single gene plays a minor role in the susceptibility to both types of diabetes. This could explain the acetylator pattern in the Type 2 group which tended towards the pattern seen in the Type 1 group but was not significantly different from normal.

There is, however, another possible explanation for the increased incidence of fast acetylators in the diabetic patients. Acetylation of drugs involves an $\mathrm{N}$ acetyltransferase reaction for which acetyl coenzyme $\mathrm{A}$ (acetyl CoA) is the acetate donor [1, 12]. It has been shown that ethanol, which increases hepatic concentrations of acetate, will increase the apparent acetylation of sulphadimidine in both fast and slow acetylators [16]. The availability of acetyl $\mathrm{CoA}$ is increased in the presence of high glucose concentrations and a preliminary study showed that isoniazid half-lives were shortened in eight healthy subjects given $100 \mathrm{~g}$ of oral glucose [17]. It might therefore be expected that in a diabetic population there would be a shift towards fast acetylation. This was the case in our population, where mean rates of acetylation were significantly higher in diabetic patients than in normal subjects for both fast and slow acetylators. Percentage acetylation was in fact a continuum amongst both Type 1 and Type 2 patients which would be expected if a biochemical factor were superimposed on a genetic background.

There was also a significant association between random, post-prandial clinic plasma glucose and acetylator status. In patients with Type 1 diabetes, $81 \%$ of those with a high plasma glucose were fast acetylators, whereas only $60 \%$ of patients with a low plasma glucose were fast acetylators which is not significantly different from the normal. Similarly, $69 \%$ of patients with Type 2 diabetes and high plasma glucose were fast acetylators compared with $40 \%$ with low plasma glucose. However Burrows et al. [8] found no association between mean fasting blood glucose and acetylator status and Bodansky et al. [9] measured glycosylated haemoglobin in their 55 Type 1 diabetic patients and failed to find an association with acetyla- tor status. These findings would be against the hypothesis of a biochemical artefact. Our glycosylated haemoglobin results also show no association with acetylator status.

The problem is a complex one. Any population will have a genetically determined proportion of fast and slow acetylators. We propose that a high blood glucose will cause a proportion of the 'slow' group to be classified as 'fast' and cause the 'fast' group to be 'faster'. A biochemically induced shift would occur after hours rather than days or weeks and therefore glycosylated haemoglobin, which reflects the previous month's mean glucose concentrations [18], is not the most appropriate way to test the hypothesis. For the same reason our retrospective glucose values can only give a suggestion of a possible biochemical artefact. The problem can only be resolved by a prospective study of acetylation in relation to changing blood glucose. It would also be of interest to measure acetylator status in discordant diabetic twins.

Meanwhile we feel that whereas acetylator phenotype may be a genetic marker for Type 1 and possibly Type 2 diabetes, it must be kept in mind that all findings published to date could be due to a biochemical artefact.

Whatever the reason for the increased incidence of fast acetylators our findings on neuropathy are at variance with those of McLaren et al. [7] and the discrepant results need an explanation. We suggest that McLaren et al. [7] may have misinterpreted their findings when they concluded that there was a higher proportion of fast acetylators in patients without neuropathy. Thus if fast acetylators have a higher than normal incidence of Type 1 but not of Type 2 diabetes and neuropathy is equally distributed between the two types of diabetes, there would be a preponderance of Type 1 fast acetylators without neuropathy. Our data tend to support this hypothesis but our numbers are too small to give a definitive answer. We consider that anything that might be a genetic marker for diabetes and its complications warrants further study but feel that at present the significance of acetylator phenotype in this regard must be viewed with caution.

Acknowledgements. We are grateful to Mrs. P. Fellowes for help with tabulating the data and to A. Maxwell-Jackson for typing the manuscript.

\section{References}

1. Evans DAP, White TA (1964) Human acetylation polymorphism. J Lab Clin Med 63:394-403

2. Lunde PM, Frislid K, Hansteen V (1977) Disease and acetylation polymorphism. Clin Pharmacokinet 2: 182-197

3. Jenne JW (1965) Partial purification and properties of the isoniazid transacetylase in human liver. Its relationship to the acetylation of para-aminosalicylic acid. J Clin Invest 44: 1992-2002 
4. Evans DAP (1965) Conjoint clinic on drugs and genes. J Chron Dis $18: 59-76$

5. Hughes HB, Biehl JP, Jones AP, Schmidt LH (1954) Metabolism of isoniazid in man as related to the occurrence of peripheral neuritis. Am Rev Tuberc Pulm Dis 70: 266-273

6. Mattila MJ, Tiitinen H (1967) The rate of isoniazid inactivation in Finnish diabetic and non-diabetic patients. Ann Med Exp Biol Fenn 45: 423-427

7. McLaren EH, Burden AC, Moorhead PJ (1977) Acetylator phenotype in diabetic neuropathy. Br Med J 2: 291-293

8. Burrows AW, Hockaday TDR, Mann JI, Taylor JG (1978) Diabetic dimorphism according to acetylator status. Br Med J 1: 208-210

9. Bodansky HJ, Drury PL, Cudworth AG, Evans DAP (1981) Acetylator phenotypes and Type 1 (insulin-dependent) diabetics with microvascular disease. Diabetes 30:907-910

10. McCann VJ, Davis RE (1979) Glycosylated haemoglobin concentrations in patients with diabetic neuropathy. Acta Diabetol Lat 16: 205-209

11. Schröder H (1972) Simplified method for determining acetylator phenotype. Br Med J 3: 506-507

12. Evans DAP (1969) An improved and simplified method of detecting the acetylator phenotype. J Med Genet 6:405-407

13. McKusick VA (1979) Mendelian inheritance in man. Catalogs of autosomal dominant, autosomal recessive and $\mathrm{x}$-linked phenotypes, 5th ed. Johns Hopkins University Press, Baltimore
14. Cudworth AG (1978) Type 1 diabetes mellitus. Diabetologia 14: $281-291$

15. Dornan J, Allan P, Elke PN, Larsen B, Farid NR (1980) Properdin factor B (Bf) allele BfFl specifies an HLA-B18 diabetogenic haplotype. Diabetes 29:423-427

16. Olsen H, Morland $\mathrm{J}(1978)$ Ethanol-induced increase in drug acetylation in men and isolated rat-liver cells. $\mathrm{Br}$ Med J 2: $1260-1262$

17. Thom S, Farrow PR, Santoso B, Alberti KGMM, Rawlins MD (1981) Effects of oral glucose on isoniazid kinetics. Br J Clin Pharmacol II: 423

18. Stanton KG, Davis RE (1978) The relationship between the control of diabetes mellitus and circulating glycosylated haemoglobin $A_{1}$. Aust NZ J Med 8: 400-404

Received: 17 June 1981

and in revised form: 11 January 1982

Dr. G.M.Shenfield

Department of Clinical Pharmacology

Royal North Shore Hospital

St. Leonards, NSW, 2065, Australia 